\title{
A pilot study of the meta-play method: a novel play intervention for toddlers with autism
}

\author{
Cooper R. Woodard ${ }^{1,2^{*}}$, Jin Chung ${ }^{2}$ and Maggie Korn ${ }^{2}$ \\ *Correspondence: cwoodard@grodencenter.org \\ CrossMark \\ ₹Click for updates
}

'The Groden Center, 86 Mt Hope Ave, Providence, RI, USA.

${ }^{2}$ University of Rhode Island, Kingston, USA.

\begin{abstract}
The goal of this pilot study was to determine if interventions based on the Dynamic Behavior Theory of Autism (DBTA) could be generated. We selected one toddler with autism and confirmed the diagnosis with the Autism Diagnostic Observation Scale (ADOS), and created a set of interventions derived from this theory. Parents and family members were trained on sets of interventions in the initial two months of research, and these interventions were added to and revised as the study progressed for an additional four month period. Each month, researchers reviewed the integrity of interventions at once-or twice-monthly home visits, and recorded progress on a variety of measures. At the end of six months, the toddler was re-tested on the ADOS. Our results suggest that we were able to create interventions based on DBTA and while no causal relations can be reasonably put forth, improvements on outcome measures including the ADOS were documented. Limitations of the current research are discussed.
\end{abstract}

Keywords: Autism, early intervention, play therapy, behavioral intervention

\section{Introduction}

Autism Spectrum Disorder (ASD) is a disorder that is characterized by the emergence of impairments in communication skills, social relatedness, and the presence of restricted or stereotyped behaviors and areas of interest prior to age 3 . It is currently estimated that 1 in 88 children are identified as having an ASD [2], and in addition to the emotional toll on the families of a person with an ASD, the increasing rates of prevalence have recently been estimated to cost the United States 35 billion dollars per year [5]. There is no single factor that has been identified as causal for autism, and its origins remain unknown despite extensive research efforts [6]. Given the early emergence of this increasingly prevalent and costly problem, identifying effective treatments models focused on early interventions, parent interactions, and structured play is important. Several models based on different theoretical orientations have been developed, including the Early Start Denver Model (ESDM) and the Pivotal Response Treatment (PRT), and while these models have demonstrated beneficial outcomes in certain areas, the effect of these models on the overall severity of the core symptoms of autism has not been demonstrated. We will briefly review these two models and relevant research outcomes, and then describe an alternate theoretical orientation that we think may have merit as it is developed into an applied treatment model.

The ESDM is a behavioral intervention set designed to improve the severity of autism symptoms and accelerate cognitive, social, and language functioning in toddlers diagnosed with an ASD. Based on research and knowledge about typical infant development, this model seeks to train parents in a style of interaction that maximizes a child's attention so that optimum parent techniques can help to improve language, social and symbolic play and social interactions [16]. Integrating Applied Behavior Analysis (ABA) with developmental and relationshipbased approaches, the core components of this model include 1) use of an interdisciplinary team to deliver a comprehensive developmental curriculum; 2) a focus on interpersonal engagement; 3 ) a fostering of spontaneous imitation of gestures, facial movements and expressions, and object use; 4) a focus on verbal and nonverbal communication as well as cognitive aspects of play, and 5) a close partnership with parents (p. 15). These components are delivered through practices such as establishing functional learning objectives, task-analyses or "learning steps," quantitative tracking of progress, teaching parents to become active play partners and learn social routines and roles in play, establishing and implementing effective reinforcers, and fostering imitation and others. The following study suggests that despite its well-formed theoretical groundwork, 
Woodard et al. Journal of Autism 2014,

implementation of this model has not resulted in significant reductions in severity of autism symptoms as measured by the Autism Diagnostic Observation Scale [12].

Dawson et al., used a randomized, controlled trial to evaluate the efficacy of ESDM. 48 children diagnosed with an ASD between the ages of 18 and 30 months were randomly assigned to receive either 1) an ESDM intervention for two years, or 2) a community provider-based intervention. Results suggested that children who received ESDM supports showed significant improvements in cognitive development, adaptive behavior, as well as additional changes in their diagnostic status. At baseline, in the ESDM group 21 children were diagnosed with Autistic Disorder and 3 were diagnosed with PDD-NOS, and in the control group 18 were diagnosed with Autistic Disorder and 6 with PDD-NOS. At the end of two years of treatment, 15 children (62.5\%) in the ESDM group retained the same diagnoses as did 15 children $(71.4 \%)$ in the control group. Seven children (29.2\%) in the ESDM group had their diagnosis changed from Autistic Disorder to PDD-NOS as compared to $1(4.8 \%)$ in the control group. However, for $2(8.3 \%)$ of the children in the ESDM group and 5 (23.8\%) of children in the control group, the diagnosis changed from PDD-NOS to Autistic Disorder. While there were further, significant improvements in IQ and adaptive behavior, there were no significant changes in ADOS Severity Scores for children in either group following one or two years of participation in the research study.

Another widely used intervention approach for young children with autism is Pivotal Response Treatment (PRT). This treatment method is based on the notion that enhancing behaviors unique to five pivotal areas of development including motivation, responsivity to multiple cues, selfmanagement, empathy, and child self-initiations will result in rapid and broad intervention outcomes [8]. Like ESDM, PRT utilizes developmental theory of typical child development as well as principles of ABA in their approach. The goal of the PRT approach is to provide intensive early intervention focused on broad areas of behavior that is delivered across settings [8]. Specific components of the PRT approach include providing the child with a clear choice of activity or materials and taking turns, responding to multiple cues, contingent reinforcement, and reinforcing goal-directed attempts [18]. The range of behaviors and skills that PRT has been reportedly used to improve include symbolic play, sociodramatic play, peer social interaction, self-initiations, and joint attention [18]. Outcome studies have demonstrated that PRT interventions resulted in a significant improvement in areas such as functional verbal utterances during parent-child interactions $[3,13]$, and complex social behaviors [15], but as with ESDM, research on this method has not demonstrated a significant reduction in the general severity of core symptoms of autism in young children.

Although intervention models such as ESDM and PRT have made substantial contributions to the area of autism treatment in generating positive outcomes in certain areas of functioning, a lack of improvement in core autism symptoms may signal that the theoretical orientations employed are somehow insufficient. A theory emerged in 2011 that suggested a significantly different theoretical origin of autism [23], although a treatment model based on this theory has not been created. This alternate theoretical perspective, later referred to as 'Dynamic Behavior Theory of Autism' (DBTA) [21], suggested that the many varied symptoms and presentations of autism are the result of the cascading effects of impaired meta-representational and imagination-based abilities early in life. This core cognitive ability is proposed to be primary to, foundational and necessary for the acquisition of many of the skill areas focused on in ESDM and PRT, which may partly explain why these models have not demonstrated improvement in the core autism features. A full discussion of the theory and its origins is documented elsewhere $[\mathbf{2 1 , 2 2 ]}$.

Briefly, DBTA suggests that autism is the result of an atypical or altered cognitive trajectory during a key period in young children's development. This key period is marked by a dramatic shift in cognition that typically takes place around a child's first birthday, and has been dubbed the "9-month revolution" by developmental scientists (e.g., [20]). Successful navigation of this shift allows the infant to not only think but "think about," opening the door to a variety of more sophisticated behavioral repertoires. This commonly accepted and important evolution in thinking skills has been identified in various ways by numerous researchers, including [19] "intersubjective perspective," [9] "meta-representation," and [24] "recursive consciousness." Regardless of the name given (we will use "meta-representation"), this more sophisticated form of thinking allows a range of important behaviors to emerge, including pointing, showing, joint attention, deferred imitation, embarrassment and coy behavior, and functional play [23]. Further, it is suggested to lay the imagination-based, cognitive groundwork for later emergence of abilities such as language, symbolic and pretend play, the ability to think about other's thinking (or "Theory of Mind"), and even much later references to the self as an objective entity. This ability to internally represent and think about ideas and images (or "meta-represent") has been noted by other authors to be "crucial" to autism during early development [1]. Simply, DBTA focuses on the 9-month revolution in thinking, and suggests that autism is a failure of or impairment in the emergence of cognitive skills that typically develop at this time.

We suggest that the behavioral markers noted above (pointing, joint attention, etc.) signal that this cognitive shift has successfully taken place, and the absence of these behavioral markers is diagnostic for autism. Further, the timing of this cognitive shift slightly pre-dates the typical initial emergence of autism. Given these facts, one might take an interest in the proposed mechanisms that fuel the "9-month revolution" in thinking when considering how to effectively intervene with a young child found to be on the 
autism spectrum. In DBTA, Woodard and Van Reet [23] built upon and extended Hobson's [7] concept of identification which suggested that the mechanism for the emergence of typical meta-representational thinking derives from the infant's experience of another whole-human person expanding, which in turn increases the awareness that other people are connected with objects and events in the world. Woodard and Van Reet extended this line of thought and suggested that the mechanism active in the emergence of autism (and impairing typical meta-representational thinking) is derived from early identification with (non-human) objects and part-objects, as contrasted with people. In other words, DBTA suggests that early inanimate (non-human) object and part-object identification (as contrasted with human identification) impairs the trajectory of typical meta-representational thinking, leading to cascading effects across the lifespan that are represented in the many varied and changing symptoms of autism.

With these concepts in mind, the primary goal of the present study was to determine if play interventions could be generated based on the concepts of DBTA, determine if parents could implement these interventions, and record any changes specific to core autism features. As will be described in detail below, the play interventions that became the "Meta-play Method" were extrapolated from DBTA in this way: The play interventions were designed to 1) shift attention and identification from objects to people, 2) engage the child in activities that fostered any type of imagination or meta-representational thinking, 3) foster tolerance for unpredictability as compared to object constancy, or 4) increase understanding of "wholes" versus "parts." Some of the activities met more than one of these theory-based criteria, and combined for example, human focus and "whole" understanding. Further, given the long history of demonstrated effectiveness of a wide array of behavioral principles, we chose to combine the conceptual core of DBTA with a wide range of behavioral strategies to produce our treatment approach. The first two months of this study consisted of 1) a pre-intervention assessment of the toddler selected, 2) creation of play interventions based on DBTA, and 3) parent training on the core concepts of DBTA, associated behavioral strategies, and the identified play interventions. This was followed by four months of once- or twice-monthly fidelity checks/home visits, which also resulted in revision of existing activities and adding to our play activity set. At the end of the total 6-month period, we repeated the assessment procedures administered at the pre-intervention stage.

\section{Methods}

\section{Participant}

One male, Caucasian toddler took part in this study and was age two years and two months at the start of the research project. Previous to the start of the study, this child had been given the diagnosis of autism. This child was recruited from the Groden Center's Early Intervention (EI) program, and his parent signed consent as approved by the Groden Center's
Institutional Review Board (IRB). He continued to receive two hours weekly of early intervention services from this organization during the time of the study, and was specifically selected because of a relatively high activity level and his obvious interest in objects. Both parents lived in the home, and this child had one older female sibling and one older male sibling. The family lived in an average, middle-class home in southern Rhode Island.

The participant was assessed by a trained clinician using the Autism Diagnostic Observation Schedule-Module 1 (ADOS; [10]), and these scores were transferred to an ADOS-2 [11], Module 1 Algorithm as this format generated the most recent autism cut-off guidelines as well as comparison/severity scores. The participant's Social Affect (SA) and Restricted and Repetitive Behavior (RRB) combined scores (Total score $=22$ ) exceeded the cut-off score for autism, as scored by a clinician unfamiliar with the present study. His combined score was consistent with a comparison/severity score of 8 , indicating a high level of autism-related symptoms. The parents also completed a standard assessment to determine what were effective reinforcers for the participant. He was on no prescription medication, was in generally good health, and had no other documented psychiatric or medical disorders.

In addition to being used to confirm the diagnosis of autism for our participant at the start of this research project, we also re-assessed the participant using the Autism Diagnostic Observation Schedule (ADOS; [10]) at the end of the six months of intervention.

\section{Measures}

\section{Autism diagnostic observation scale (ADOS)}

The ADOS is currently the gold-standard for the identification of symptoms related to autism, and has four separate modules. Our participant took part in module one, which consists of structured and standardized play situations. This scale is widely used and is a well-validated research measure for current severity of autistic impairment. A new version of the ADOS was published shortly after we initiated our research study (ADOS-2), and because this version contained the most recent guidelines of autism cut-off scores and comparison/ severity scores, the algorithms from the ADOS-2 were used for the present research study.

\section{Clinical global impressions scale (CGIS)}

A modified version of the Clinical Global Impressions Scale (CGIS) $[14,17]$ was used and completed monthly by the participant's parents. This version of the CGIS is a brief seven-item questionnaire reflecting the core and associated symptoms of autism. It is adapted for this use from the Clinical Global Impressions Scale and uses a 1 (very much improved) to 7 (very much worse) rating scale. For this study, the 7-point CGIS rating scale was used to record ratings in each of the seven symptom areas related to autism (behavior problems, responds to social interactions, initiates social interactions, use of speech to commu- 
Woodard et al. Journal of Autism 2014,

http://www.hoajonline.com/journals/pdf/2054-992X-1-3.pdf

doi: 10.7243/2054-992X-1-3

nicate, repetitive behaviors, activity level, and nighttime sleep).

\section{Parent activity log}

The parents were given a log to complete daily which listed the activities that had been created by the researcher. This log included instructions for the parents on how to correctly implement the activities, which is represented in the listing of activities in a slightly different format below. The list was added to as additional interventions were created in the initial two months of the study, and had a total of 12 items at the end of the research project. Alterations to the explanations of activity descriptions were made throughout the study, and the parents were encouraged to suggest alterations to activities so the researchers could determine if these were consistent with the original concepts. Each item had a description of the activity phrased in simple language, and a section in which the parent could identify how long he or she was able to implement the intervention. This was followed by a section where the parent could note the child's response, and any general comments.

\section{Procedure}

This study was reviewed and approved by the Groden Center Institutional Review Board (IRB), and the parents agreed to participate by signing an informed consent. Following the autism-specific and reinforcer assessments noted above, the researchers gave the parents the original article that formed the basis for DBTA [23], and met with the parents to ensure a level of understanding. The purpose of explaining the theoretical basis for the intervention was so the parents could potentially augment the intervention set generated by the researchers, as well as understand the purpose of the activities. The procedure that followed was comprised of three main components, which included 1) the creation of the intervention set based on DBTA, 2) parent training on these interventions and associated behavioral principles, and 3) monitoring of implementation of the intervention set and revisions to the interventions. Finally, the participant was re-assessed for autism-specific symptoms. Each of these components will be discussed in turn in the following sections.

\section{Creation of intervention set based on DBTA}

To create the activities or interventions, the researchers used as guides the main concepts central to DBTA. There were four main concepts identified from this theory which were relevant to generating a method of intervention:

1. Any process of imagination from object existence to the more abstract human imagining.

2. Any engagement that is out of the child's control or a product of (human) unpredictability vs. object predictability.

3. Any activity that fosters movement on the object to human continuum.

4. Any activity that fosters movement on the part to whole continuum.

Using these concepts, the researchers developed a set of activities or interventions and referred to this set as the "Meta-play Method." A brief description of each activity was created for parent reference as follows:

\section{Pairing reality with representation (PRR)}

Pairing in natural contexts increasingly "symbolic" objects with real objects, or engaging in same activities with your child, and then the doll engages in the activity with the child in a parallel way. For example, when you use a real cup or a phone, have the child use a toy cup (and if he or she does this, move on to a cylinder) or use a toy phone.

\section{Fostering imagined existence (FIE)}

Training a behavior that fosters object permanence thinking, beginning with shaded boxes hiding objects and progressing as noted below. Reinforce any spontaneous searching for anything hidden. The goal is to have the child imagine that you could have put the hidden object or person anywhere, and for him or her to imagine possibilities of location.

Sequence of this activity begins with the non-shaded box, then the partially shaded box, and then the fully shaded box. When the child can do this last box, move on to the child being able to see the object be hidden elsewhere in the room. Then let the child see you hide somewhere, and let him or her find you in the same spot repeatedly. Next, try hide and seek in various locations, reinforcing the child when he or she finds you. Finally, try getting the child to hide and you find the child. (The progression is from object to human, and from the child imagining objects to imagining your thinking).

\section{Practice unpredictable/other-controlled play (PUP)}

Parent removes control and predictability in short to long periods with reinforcement, such as unpredictable toys such as non-directional balls or remote control cars or swinging/ flying the child.

\section{Restricted parallel animal/people/puppet play (RAP)}

Exposure to approximations of people/animal only + puppets/ humanoid objects (to the exclusion of any other object), such as Thomas the Train, puppet play, or objects with human trait play/animals with human traits.

\section{Reinforced putting people together (PPT)}

Play that involves assembly of human/animal/human-like toys, such as Mr. Potato Head, the pop-up toy or the bear or doll in pieces. Begin with whatever the child is interested in and will do, and when the child clearly can take part in this behavior, have him or her put together a picture of the self. Reinforce the behavior.

\section{Incidental entertainment training (IET)}

Videos where objects are people or have human traits, such as Beauty and the Beast, Aladdin, Cars, or Thomas the Train. 


\section{Parallel Puppet play (PPP)}

Start by using object puppets that engage in what should appear to the child to be "joint attention" or interactive play, and then move on to animal or part-human, and then human puppets that do the same thing. If the child hits or pushes the puppet away, pause with the puppet out of reach, and then restart the interaction.

\section{What's in the box? (WIB)}

Using a box with holes in it (big enough on one side for the child's arm and big enough on the other side for you to place items), shape getting things out of the box. Move in the progression as indicated, and finally ask the child to select one item from a choice of three. Move from object to more human.

\section{Acting on other's intent (AOI)}

Demonstrate a need when you have the child's attention, and then use prompt fading from physical to gestural to no prompt. For example, needing or planning to open the door, eat something, or grab something.

\section{Object to person video-modeling (OPV)}

Have the child watch videos that have been made for you and are all from the child's perspective. These will begin with looking from objects to faces, responding to name, joint attention with a parent, and then watching a person and their facial expression.

\section{Most human card game (MHC)}

Choosing the most human card out of an array of 3, starting with two distractors.

\section{Pairing reality with representation 2 (PRR-2)}

Matching real objects to symbol cards with 2 distractors: Have the child match first a real cup to a picture of a cup (out of a group (array) of three items with two being distracters, and the instruction of "Match"), and then a cup to a picture of a toy cup with the same number of distracters; phone to picture of a real phone (out of a group (array) of three items with two being distracters), and then phone to picture of toy phone.

Because each of these activities was implemented by the parents in stages, a more detailed chart that described the levels of each intervention was created and is contained in Supplement Table. A complete listing and discussion of these activities are described in "The Meta-play Method: Application of the Dynamic Behavior Theory of Autism" [21], and "The Metaplay Manual: Theory-based Interventions for Young Children with Autism" [22].

Parent training on interventions and behavioral principles In the initial two months of the research project, the created activities that came to be known as the "Meta-play Method" were introduced to the parents and reviewed for clarity. In regular home visits, the researchers asked the parents to demonstrate the implementation of the activities with the participant/toddler when he would cooperate, or at least describe and demonstrate the activities with the researcher. It is notable that initially, many of the activities were met with tantrum behavior or other forms of refusal, so the researchers encouraged the parents to continue to shape the desired behaviors step by step and instructions were altered accordingly. The researchers were also available by email and phone to answer questions, and the parents made good use of these communication options. For example, for the FIE activity, the parents contacted the researchers because the toddler was searching for others, but would not hide. We suggested to the parents that the toddler be paired with his sister to practice hiding before he was expected to do this on his own, which was a practice that was later integrated into the intervention set. Most of the activities were created in the initial two months of the research project, and the parents were implementing them regularly at the end of this period. The exception to this was any activity in discrete trial format using two-dimensional materials, which was not a form of intervention in which this child showed interest.

In addition to the intervention set, the parents were trained on a variety of behavioral interventions and strategies that were found to be particularly relevant to implementation of the Meta-play Method activities. These included basic concepts such as shaping behaviors (progressively based on the information in Supplement Table), how to identify reinforcers and effective use of reinforcement, prompting, extinction of non-desired behavioral repertoires, and the antecedentbehavior-consequence contingency set. Additional concepts that were presented included the importance of recording data accurately and repeated practice of interventions, and gaining attention prior to beginning an activity. As the intervention set became more familiar to the parents, additional concepts became relevant and were added to training, such as generalization. Training on these behavioral strategies gave the parents and researchers a terminology with which to communicate, and complemented implementation of the intervention set.

\section{Monitoring of implementation}

In the four months that followed this 2-month introductory training period, the researchers came out to the home either once or twice per month to check on the child's progress, assess fidelity of interventions, and answer any questions or issues that arose. The parent regularly completed the Parent Activity Log on a daily basis, which recorded the approximate daily frequency with which the child was engaging in the identified activities and other information noted above. When the researchers came to the home, the researchers engaged in the activities with the toddler to model appropriate implementation of the intervention set, and also had family members engage in the activities to demonstrate fidelity. At the close of the sixth month, the parent brought the toddler in for re- 
Woodard et al. Journal of Autism 2014,

http://www.hoajonline.com/journals/pdf/2054-992X-1-3.pdf

doi: 10.7243/2054-992X-1-3

testing on the ADOS, and for a closing interview.

\section{Results}

Narrative progress as reported by parents

Our experience with the participant and his family demonstrated that activities or interventions based on DBTA and supported by known behavioral strategies could be created and trained by the researchers, and implemented by parents. While the child did not take part in every activity, there were many that he did take part in actively and came to enjoy. Certain activities needed to be adapted based on the child's likes, dislikes, and interests, and all needed to be attempted when the child was willing and ready. The parents of this particular child remained positive and interested in the project, and as a result were able to adapt certain activities in creative ways. They understood both the central DBTA concepts and the supplemental behavioral concepts, and created parallel activities in varied contexts that interested the child. For example, the child was interested in steering, so the parents used a toy version in the car so he could pretend to 'steer' in manner consistent with the parent's true steering. The parents were also adept at knowing when and when not to attempt to gain cooperation from the child, and showed tremendous patience in slowly exposing the child to less desired activities and shaping desired outcomes. Another advantage of this particular family was the presence of two older siblings who were eager to implement the activities, and patient with the child's setbacks and problem behaviors.

A review of the parent activity logs showed that the parents completed the logs approximately $82 \%$ of the days during the first 4.5 months of the study. Each day, the parents recorded that 1 to 5 of the activities were conducted with the participant, and the number and variations of the activities increased during the study period. The parents commented on the activities as they were able, and progression on the various activities was apparent. For example, for the FIE activity described above, the participant progressed through use of the boxes quickly, and moved on to further challenges as suggested. He initially appeared to be seeking to find objects, and never actually "found" anyone when people hid. Then, he only sought out others who were hiding or familiar objects that were hidden; the parents then generalized the "seeking game" to the park environment, and added a seeking "elf on the shelf" game during the holiday season. By the end of month 2, the participant was actively searching for objects in unique locations, and for the first time in month 3 , hid with assistance. Initially, he did not enjoy being found, but by month 4, the participant was hiding more easily and reliably, and this behavior continued through month 5 .

The parent activity log also shows a general improvement in other areas related to the participant's diagnosis. Early in treatment, the participant resisted others playing with him, had severe sleep problems, and demonstrated tantrums with marked aggression when engaged with certain study activi- ties or other activities. By month 3, he began using more new words (e.g., "stop," "wet," "no"), and also began demonstrating spontaneous acts of affection mainly toward the mother. This affection generalized to the brother and father, and at the end of month 3, the participant used a 2-word phrase. Additional words emerged in month 4 , and the parent reported that the participant was "babbling." Also in month 4, the participant was noted to have played well with other children, and was taking part in a wide variety of pretend play activities.

Further, at the end of month 2, the parents reported "difficult" behaviors of grabbing and attempting to bite, about 5 words, pointing at objects in a book, and the inconsistent emergence of responding to name. As time went on during the intervention phase, there was increased engagement with parents and other family members reported, but the participant continued to display aggression when others reached for something that was his. At the end of month 3 , the parents reported more vocalization attempts, and spontaneous naming of pictures. They also reported that the participant imitated the mother cleaning a surface, as well as her smelling a flower. Pointing became consistent and generalized to foods that he wanted. Responding to name became more robust, and the parents reported that the participant stopped secluding himself in his room.

During month 4, the parents reported more "babbling" consistent with the activity log, and began saying "thanks" and engaging others in a "knock-knock" game. He was noted to be playing more cooperatively with others, and was "driving" on the play steering wheel in the car in symbolic-toy-imitation of his mother. Aggression continued to decrease, and the participant tolerated activities such as bath-time better. By month 5, the participant was saying "no" to a variety of nondesired activities, such as brushing his teeth, and engaging cooperatively in group activities at his El program. Two-word phrases became more common, and the participant verbalized "help mom," "big bubbles," and "not now." He continued to imitate the mother's actions in a variety of settings, and was described as happy and engaging more with others generally.

\section{CGIS progress}

Result of the CGIS are rated on a scale of 1 (very much improved) to 7 (very much worse), with 4 corresponding to no change. The average ratings for months 1 to 6 were 3.6, 3.5, 3.5, 2.8, 3.1 and 2.8 respectively. These averages indicated "minimal" to "much" levels of improvement each month of the study, and are represented (Figure 1). The greatest improvements were noted in social interactions, speech, and activity level as represented by the highest frequency of " 2 " or " 3 " ratings. It is noted that in the area of social initiation, progress was minimal which was consistent with the ADOS results at the 6-month administration.

\section{Autism-specific assessment (ADOS using ADOS-2 algorithms)}

The ADOS was completed at the start of the research and 


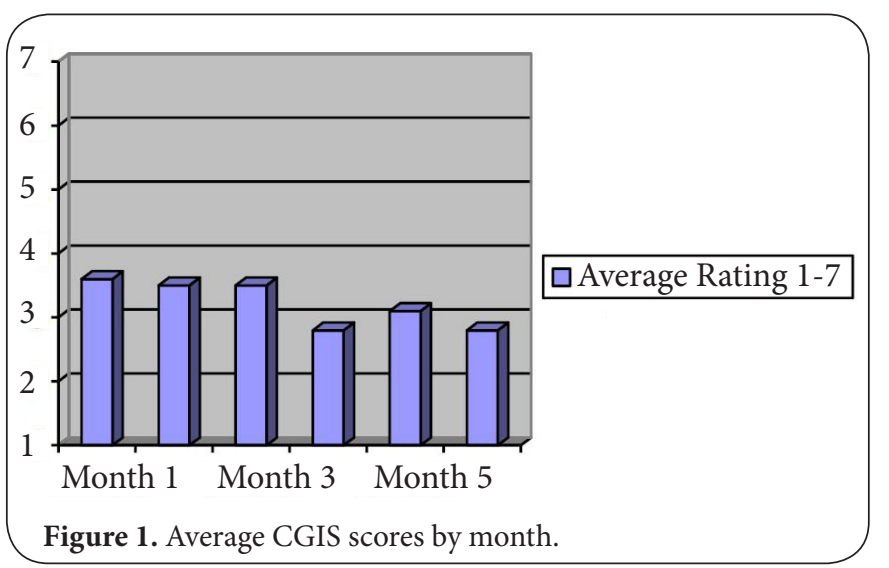

at the end of 6 months of treatment. No conclusions can be drawn from comparisons of these two pieces of data due to the many confounding variables. Some of the confounding variables include the fact that the child was receiving services from the Groden Center El program during the time of this research study, the parents became more informed and adept at using behavioral strategies, and the overall general level of engagement among family members increased dramatically during the research study. Further, the present case study was a single participant and there was no control participant for comparison. With these limitations in mind, we can report on the ADOS findings but cannot draw any conclusions. Ratings on the initial administration of the ADOS (completed by a clinician blind to the purpose of the project) which were transposed to the ADOS- 2 algorithms, indicated a Social Affect (SA) and Restricted and Repetitive Behavior (RRB) combined score of 22 as noted above, which exceeded the cut-off score for autism. This score indicated a comparison/severity score of 8 , which suggests a high level of autism-related symptoms (Figure 2).

At the second administration of the ADOS (which was also scored by a clinician blind to the project) following 6 months of our interventions, the Social Affect (SA) and Restricted and Repetitive Behavior (RRB) combined score was reduced to 7 , which no longer exceeds the cut-off scores for autism or an autism spectrum disorder. This combined score indicated a

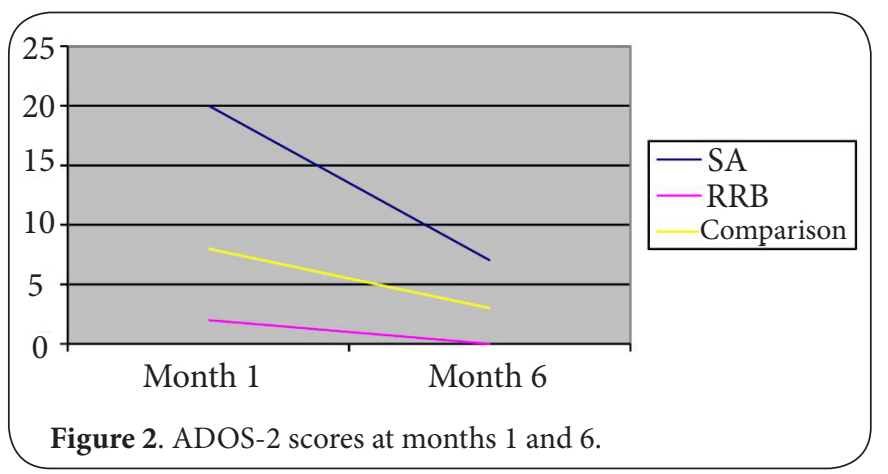

comparison/severity score of 3 , which suggests a low level of autism-related symptoms.

\section{Discussion}

A number of early intervention treatment models have been created and shown important benefits, but none have shown a direct improvement of autism-specific symptoms. We suggest that this may in part be due to these researchers not employing theoretical models that effectively identify the source of autism. A new model, the Dynamic Behavior Theory of Autism (DBTA) hypothesizes that the many varied symptoms and presentations of autism across the lifespan are the result of an altered early developmental trajectory resulting from impairment of meta-representational abilities. This impairment is hypothesized to emerge as a result of an inanimate object/part-object identification as compared to a human object/whole-object identification process. With this theoretical orientation in hand, we were able to create intervention activities that were consistent with the theory. The activities focused on transitioning from inanimate objects to human objects, part-objects to whole-objects, and any activity that also furthered meta-representational or imagination-based thinking. We combined these with proven behavioral strategies, trained parents on both components, and found that parents could implement the activities. Additionally, the toddler selected as the participant actively took part in the activities. A key component was adjusting and varying the interventions so that the toddler was attending and interested, and remained so. Once parents understood the concepts of the theory and how they were being applied, they were able to create related activities that built upon and expanded the main components and interventions set.

\section{Conclusion}

During the period of the study, the measures employed indicated minimal to moderate improvements in general areas related to functioning for the child with autism, and diminished core symptoms. None of the activities appeared to have any adverse effects on the child or the family members. While no causal relations can reasonably be drawn from this information due to a wide range of confounding variables (e.g., teaching of behavioral principles, El service delivery, consultation regarding behavioral challenges, etc.), this information is encouraging. At the very least, the sets of play activities gave the parents specific and concrete things to do with the child and provided an opportunity for learning about various complementary behavioral strategies. There are many limitations to the present research, most notably that there was only one participant involved and this participant was specially selected based on his activity level and interest in objects in his environment. Further, this child could perhaps have made improvements without any intervention or assistance at all, especially given the robust involvement of his family members. Further research is needed that provides a control-group 
Woodard et al. Journal of Autism 2014,

http://www.hoajonline.com/journals/pdf/2054-992X-1-3.pdf

doi: 10.7243/2054-992X-1-3

and a larger sample to determine if DBTA and the Meta-play Method are actually variables that affect the severity of core autism symptoms. However, we have demonstrated that activities can be developed based on this theory, and that both infants and parents can take part in them. It remains to be seen whether or not such interventions actually foster meta-representational, imagination-based thinking, and if such changes alter the emergence of the symptoms of autism.

\section{Additional files}

\section{Supplement Table}

Competing interests

The authors declare that they have no competing interests.

Authors' contributions

\begin{tabular}{|l|c|c|c|}
\hline Authors' contributions & CRW & JC & MK \\
\hline Research concept and design & $\checkmark$ & $\checkmark$ & -- \\
\hline Collection and/or assembly of data & $\checkmark$ & -- & -- \\
\hline Data analysis and interpretation & $\checkmark$ & -- & -- \\
\hline Writing the article & $\checkmark$ & $\checkmark$ & -- \\
\hline Critical revision of the article & -- & -- & $\checkmark$ \\
\hline Final approval of article & $\checkmark$ & -- & -- \\
\hline Statistical analysis & -- & -- & -- \\
\hline
\end{tabular}

Acknowledgement and funding

We would like to acknowledge the Autism Society of AmericaRhode Island Chapter for providing funding for this research.

\section{Publication history}

Editor: Larry Jarrett Barnhill, University of North Carolina at Chapel Hill, USA.

Received: 24-Jun-2014 Final Revised: 03-Aug-2014

Accepted: 19-Sep-2014 Published: 27-Sep-2014

\section{References}

1. Bernabei $P$, Cerquiglini A, Cortesi F and D'Ardia C. Regression versus no regression in the autistic disorder: developmental trajectories. J Autism Dev Disord. 2007; 37:580-8. | Article | PubMed

2. CDC Autism Report. 2010.

3. Coolican J, Smith IM and Bryson SE. Brief parent training in pivotal response treatment for preschoolers with autism. J Child Psychol Psychiatry. 2010; 51:1321-30. | Article | PubMed

4. Dawson G, Rogers S, Munson J, Smith M, Winter J, Greenson J, Donaldson $A$ and Varley J. Randomized, controlled trial of an intervention for toddlers with autism: the Early Start Denver Model. Pediatrics. 2010; 125:e17-23. | Article | PubMed

5. Ganz M. L. The costs of autism. In S.O. Molden \& J.L.R. Rubenstein (eds.). Understanding Autism: From Basic Neuroscience to Treatment. Boca Raton, FL: Taylor and Francis Group. 2006.

6. Happe F, Ronald $A$ and Plomin R. Time to give up on a single explanation for autism. Nat Neurosci. 2006; 9:1218-20. | Article | PubMed

7. Hobson P. The cradle of thought. New York: Oxford University Press. 2004.

8. Koegel R.L, Openden D, Fredeen, R and Koegel L. K. The basics of pivotal response treatment. In R. L. Koegel \& L. K. Koegel, (eds.) Pivotal response treatments for autism. Baltimore, Maryland: Paul H. Brooks Publishing Co. 2006; 4-30.
9. Leslie A. Pretense and representation: The origins of "Theory of Mind." Psychological Review. 1987; 94:412-426. | Article

10. Lord C, Risi S, Lambrecht L, Cook EH, Jr., Leventhal BL, DiLavore PC, Pickles $A$ and Rutter $M$. The autism diagnostic observation schedulegeneric: a standard measure of social and communication deficits associated with the spectrum of autism. J Autism Dev Disord. 2000; 30:205-23. | Article | PubMed

11. Lord C, Rutter M, DiLavore P.C, Risi S, Gotham K and Bishop S.L. Autism Diagnostic Observation Schedule, Second Edition (ADOS-2) Manual (Part I): Modules 1-4. Torrance, CA: Western Psychological Services. 2012.

12. Lord C, Rutter M and Le Couteur A. Autism Diagnostic Interview-Revised a revised version of a diagnostic interview for caregivers of individuals with possible pervasive developmental disorders. J Autism Dev Disord. 1994; 24:659-85. | Article | PubMed

13. Minjarez MB, Williams SE, Mercier EM and Hardan AY. Pivotal response group treatment program for parents of children with autism. J Autism Dev Disord. 2011; 41:92-101. | Article | PubMed

14. National Institute of Mental Health. CGI (Clinical Global Impression) Scale. Psychopharmacology Bulletin. 1985; 21:839-843.

15. Pierce $K$ and Schreibman L. Multiple peer use of pivotal response training to increase social behaviors of classmates with autism: results from trained and untrained peers. J Appl Behav Anal. 1997; 30:157-60. I Article | PubMed Abstract | PubMed Full Text

16. Rogers S. J and Dawson G. Early start Denver model for young children with autism. New York: The Guilford Press. 2010

17. Sandler AD, Sutton KA, DeWeese J, Girardi MA, Sheppard V and Bodfish JW. Lack of benefit of a single dose of synthetic human secretin in the treatment of autism and pervasive developmental disorder. $\mathrm{N} \mathrm{EnglJ}$ Med. 1999; 341:1801-6. | Article | PubMed

18. Stahmer A.C, Suhrheinrich J, Reed S, Bolduc C and Schreibman L. Pivotal response teaching in the classroom setting. Preventing School Failure. 2010; 54:265-274. | Article

19. Stern D. The interpersonal world of the infant. New York: Basic Books. 1985.

20. Tomasello M. The cultural origins of human cognition. Cambridge: Harvard University Press. 1999.

21. Woodard C.R. The meta-play method: Application of the dynamic behavior theory of autism. Le Vergne, TN: Lightning Source/Ingram. 2012. | Book

22. Woodard C. R. The meta-play manual: Theory-based interventions for young children with autism. Le Vergne, TN: Lightning Source/Ingram. 2012. | Book

23. Woodard CR and Van Reet J. Object identification and imagination an alternative to the meta-representational explanation of autism. $J$ Autism Dev Disord. 2011; 41:213-26. | Article | PubMed

24. Zelazo P.R. A developmental-behavioral perspective on early autism: Affective, behavioral, and cognitive factors. In J. A. Burack, T. Charman, N. Yirmiya, \& P. R. Zelazo (Eds.), The development of autism: Perspectives from theory and research Mahwah,NJ: Lawrence Erlbaum Associates. 2001; 39-60.

Citation:

Woodard CR, Chung J and Korn M. A pilot study of the meta-play method: a novel play intervention for toddlers with autism. J Autism. 2014; 1:3. http://dx.doi.org/10.7243/2054-992X-1-3 